\title{
Usare strumenti empirici per risolvere problemi: indagine nella scuola secondaria
}

\section{Giorgio Asquini - Francesca Marano*}

Sapienza Università di Roma - Department of Developmental Psychology and Socialisation (Italy)

doi: 10.7358/ecps-2016-014-asqu

giorgio.asquini@uniroma1.it maranofra@tiscali.it

\section{USING EMPIRICAL TOOLS TO SOLVE PROBLEMS: A SURVEY IN SECONDARY SCHOOL}

\section{Abstract}

The paper presents a deepening of survey problem solving and geographic skills. How to evaluate the competence in the three years of lower secondary school. A second survey was also conducted, after the main investigation with the same tools, in upper secondary school (10th grade), then the data of the two populations are comparable. The deepening aims to investigate the relationship between the use of empirical tools to measure distances on maps and performance in problem solving. The problem solving model used by OECD-PISA is presented, also in relation to its potential educational applications. The analysis of the data confirms a positive correlation between the use of tools and performance for students of lower secondary school and no gender differences. The results for upper secondary school is more complex: the use of some tools does not affect the performance; there are gender differences in performance by the number of tools used.

Keywords: Empirical Tools, Geography, OECD-PISA, Problem solving, Secondary School.

* Il saggio è frutto di un lavoro comune degli autori. Tuttavia la scrittura dei paragrafi è così attribuita: Giorgio Asquini: $\$ \$ 1,2,3,3.1,4$ e 4.3; Francesca Marano: $\$ \$$ 3.2, 4.1 e 4.2. Il $\$ 5$ è attribuito a entrambi gli autori. 


\section{INTRODUZIONE}

C'è un recente risultato italiano nelle indagini comparative internazionali che non è stato affrontato in modo adeguato nel dibattito pedagogico nazionale. Parliamo dell'ottima, quanto inattesa, performance dei nostri studenti quindicenni nel problem solving in PISA 2012 (Asquini, 2014b). Eppure la capacità di risolvere situazioni problematiche è da diversi decenni riconosciuta come una delle abilità più utili per affrontare qualsiasi situazione, da quelle di studio a quelle della vita quotidiana. Scoprire dai dati PISA che si tratta di uno dei (pochi) punti di forza dei nostri studenti dovrebbe essere solo il primo passo per riflettere sulle motivazioni di questo risultato e sulle modalità per trasferire questa abilità agli altri ambiti disciplinari. Quando l'OCSE nel 2003 ha inserito il problem solving nell'indagine PISA (OECD, 2004; Asquini, 2006), di fatto ha concretizzato un'idea che circolava da tempo fra gli esperti di valutazione: superare le tradizionali prove disciplinari, indagando alcune abilità comuni, definite anche come trasversali alle discipline. In questa direzione andava anche il dibattito seguito alle intuizioni di Gardner circa le intelligenze multiple (Gardner, 1983), tra le quali ricordiamo l'intelligenza visuo-spaziale, con la necessità di ampliare il quadro valutativo con compiti e prove che indagassero aspetti diversi dalle semplici conoscenze o abilità pratiche. Questo non vuol dire penalizzare le discipline (anche in PISA il problem solving si è affiancato e non ha sostituito i tradizionali ambiti), quanto piuttosto fornire un nuovo punto di vista che, partendo dalla valutazione, mira a stimolare la didattica, utilizzando la proposizione di situazioni problematiche da risolvere come strumento utile in tutti i contesti disciplinari. Fino a qui il dibattito internazionale e la conseguente decisione dell'OCSE. Nel nostro paese poco è successo, anche dopo gli inattesi risultati di PISA 2012, anche se, come vedremo, il problem solving è chiaramente indicato fra le otto competenze chiave di cittadinanza anche nella normativa scolastica nazionale.

Per fornire un contributo utile al dibattito pedagogico nazionale è stato realizzato, grazie al finanziamento di ateneo della Sapienza, Università di Roma, lo studio "Problem solving e abilità geografiche. Come valutare la competenza nel triennio della scuola secondaria di I grado», con l'obiettivo di sperimentare e validare un pacchetto di prove su una specifica area del problem solving, quella legata alla risoluzioni di situazioni problematiche di tipo geografico. 


\section{L'ARTE DI RISOLVERE PROBLEMI}

Un impulso determinante ad un approccio didattico al problem solving è stato quello fornito da Polya (1945), che, partendo dalla sua esperienza di studio nel campo matematico sottotitola il volumetto How to solve it con $A$ system of thinking which can help you solve any problem, indirizzando esplicitamente a tutti gli insegnanti. Il processo di risoluzione in quattro fasi definito da Polya è tuttora validissimo, ed ha anche ispirato il modello di valutazione del problem solving di PISA (OECD, 2003, pp. 170-171), come possiamo vedere nella Tabella 1 .

Tabella 1. - Confronto fra i modelli di Polya e di PISA per il problem solving.

\begin{tabular}{ll}
\hline LE FASI DI POLYA & I PROCESSI COGNITIVI DI PISA \\
\hline Comprendere il problema & Comprendere il problema \\
& Individuare le caratteristiche del problema \\
Fare un piano & Costruire una rappresentazione del problema \\
Attuare il piano & Risolvere il problema \\
Riflettere e valutare & Riflettere sulla soluzione \\
& Comunicare la soluzione \\
\hline
\end{tabular}

Il parallelismo è evidente, con il processo «Individuare le caratteristiche del problema» che specifica e unisce le fasi della comprensione e della progettazione definite da Polya. Analizzando la descrizione puntuale dei processi di PISA bisogna però ricordare che il processo "Risolvere il problema» contiene anche elementi di selezione della soluzione, in relazione ai possibili diversi tipi di problema identificati nei processi precedenti, rimarcando che la progettazione non è un processo finito, ma prosegue nel corso di tutta la fase attuativa attraverso l'analisi di elementi che via via possono emergere. Da sottolineare infine anche la peculiarità dell'ultimo processo di PISA, legato alla necessità di costruire prove di valutazione, cioè sulla capacità degli studenti di rispondere a domande per comunicare come risolvere la situazione problematica.

Naturalmente lo spunto di Polya è stato ripreso e ampliato da molti autori, che hanno approfondito i meccanismi del problem solving in relazione alla sua spendibilità didattica. Mayer (1992) ha sottolineato come una efficace didattica debba proporre situazione problematiche non scontate, quindi non basate su automatismi, ma che necessitano di una riflessione per scegliere fra diverse opzioni disponibili, valutandone in corso d'opera l'efficacia risolutiva. Lo stesso Mayer (1998) ha anche sottolineato che l'approccio per 
problemi rinforza la motivazione a rispondere, sollecitando in maniera positiva quindi gli aspetti cognitivi e spingendo il risolutore ad accettare la sfida per vincerla, ovvero per trovare la soluzione. Anche Bransford, Brown, \& Cockling, 1999, e Novick \& Bassok, 2005, hanno molto insistito sulla spendibilità scolastica di un approccio per problemi, sottolineando come la scuola possa raccogliere dalle situazioni concrete che si prospettano ogni giorno agli studenti, nella scuola come nella loro esperienza quotidiana, spunti utili per mettere alla prova quanto imparato nel corso del percorso di studio.

Le sollecitazioni provenienti dalla ricerca in questo caso sono state colte prontamente dai decisori politici, a cominciare dal progetto De.Se.Co. promosso dall'OCSE (Definition and selection of key competencies: Ryjchen \& Salganik, 2000), in cui si sottolinea l'azione riflessiva come principale chiave di lettura per affrontare l'istruzione, e il problem solving rappresenta chiaramente una delle principali competenze chiave da promuovere proprio per la sua capacità di tenere insieme azione e pensiero, sollecitando la riflessione a partire da situazioni reali. In particolare, sono due le categorie direttamente interessate, "Agire in autonomia» e "Usare strumenti in modo interattivo". Ma come sta cercando di fare, anche PISA (OECD, 2013b), con non pochi problemi per passare alla fase operativa, attraverso la proposizione di prove di problem solving collaborativo, anche la terza dimensione (interagire in gruppi eterogenei) può essere perseguita tramite una didattica per problemi. D'altra parte PISA fin dal primo ciclo di PISA 2000, quindi prima ancora che il problem solving diventasse ambito di rilevazione autonomo, ha costruito molte delle sue prove come problemi da risolvere sfruttando dati e informazioni diverse, non solo in campo matematico (OECD, 1999), e parallelamente ha inserito la risoluzione di problemi anche nelle rilevazioni sugli adulti (Statistics Canada \& OECD, 2005), confermandola anche nella più recente indagine PIAAC (OECD, 2016). Risulta però essenziale riportare la prima definizione ufficiale del problem solving formulata da PISA per la rilevazione del 2003.

Per Problem Solving si intende la capacità di un individuo di mettere in atto processi cognitivi per affrontare e risolvere situazioni reali e interdisciplinari, per le quali il percorso di soluzione non è immediatamente evidente e nelle quali gli ambiti di competenza o le aree curricolari che si possono applicare non sono all'interno dei singoli ambiti della matematica, delle scienze o della lettura. (Asquini, 2006, p. 92)

I processi cognitivi indicati fanno riferimento a diversi tipi di modi di pensare che l'individuo, di fronte a una situazione problematica, deve sapere estrarre dal proprio repertorio intellettivo e padroneggiare. Si va dal ragionamento di tipo analitico, che si basa su elementi logici, al ragionamento quantitativo, che considera dati di tipo numerico, al ragionamento analogico, che rielabo- 
ra casi simili precedenti per verificare la possibile trasferibilità di soluzioni già utilizzate, al ragionamento combinatorio, che mette insieme in modo flessibile il maggior numero di dati disponibili utili per la strategia risolutiva (De Young, Flanders, \& Peterson, 2008).

Una efficace didattica basata sul problem solving dovrebbe quindi da una parte sollecitare la più ampia gamma di processi cognitivi, dall'altra insistere sull'applicazione del modello per fasi visto in precedenza, e questo è possibile solo proponendo contesti problematici originali, non risolvibili con procedure standard di routine, ma affrontabili tramite la costruzione di una strategia creativa di soluzione (Jonassen, 2011).

La strada aperta dal progetto De.Se.Co. è stata proseguita con importanti documenti politici di orientamento per le scelte educative. A livello europeo nel 2006 è stato definito un documento sulle competenze chiave di cittadinanza, con relativo invito a tutti i paesi membri di aggiornare in tal senso la loro normativa. L'Italia ha avviato il percorso quasi subito, con il D.M. 139/2007, che nell'allegato 2 inserisce come sesta competenza chiave di cittadinanza «Risolvere problemi», con la seguente definizione:

Affrontare situazioni problematiche costruendo e verificando ipotesi, individuando le fonti e le risorse adeguate, raccogliendo e valutando i dati, proponendo soluzioni utilizzando, secondo il tipo di problema, contenuti e metodi delle diverse discipline.

La conclusione marca in modo chiaro il carattere trasversale a tutte le discipline del problem solving, quindi non si può dire che manchi, a livello normativo, un invito chiaro ad agire nella pratica quotidiana sfruttando le potenzialità formative di un approccio per problemi. L'evoluzione del problem solving in PISA è partita quindi da un approccio analitico, per evolversi in una prospettiva interattiva e mirare in futuro anche alla dimensione collaborativa (Greiff, Holt, \& Funke, 2013).

\section{LA RISOLUZIONE DI SITUAZIONI PROBLEMATICHE IN AMBITO GEOGRAFICO}

Alla luce dell'importanza riconosciuta al problem solving, sia dalla ricerca educativa, sia dalla normativa più recente, è stato avviato lo studio "Problem solving e abilità geografiche. Come valutare la competenza nel triennio della scuola secondaria di I grado»" ${ }^{1}$ con l'obiettivo principale di verificare l'esisten-

1 Il progetto di ricerca è stato realizzato tra il 2012 e il 2015, grazie al finanziamento di ateneo 2011 dell'Università Sapienza di Roma. 
za di possibili correlazioni fra la capacità di risoluzione dei problemi e diversi fattori scolastici e personali. Nella costruzione della prova per rilevare la capacità di problem solving si è deciso di delimitare il campo d'indagine dal punto di vista dei contenuti, considerando i diversi vincoli di ricerca, proponendo solo situazioni problematiche relative all'ambito geografico. Per rilevare le variabili di sfondo è stato predisposto un questionario studente, strutturato su diversi aspetti: elementi anagrafici, modalità personali di studio e di approccio alle situazioni problematiche, esperienze didattiche nel corso della carriera di studio, utilizzazione delle tecnologie, conoscenza e pratica dell'ambito geografico, prospettive di studio.

\subsection{Perché il problem solving geografico}

La scelta di specificare fortemente il contesto della prova è nata anche dalla constatazione di una sostanziale mancanza di studi specifici, anche se nelle prove proposte da PISA nel ciclo 2003 alcune erano basate su problemi di movimento nello spazio (OECD, 2004; Asquini, 2006). La costruzione delle prove si è basata proprio sul quadro di riferimento di PISA 2003, già richiamato nel paragrafo precedente (OECD, 2003), considerando che, nella fase di allestimento delle prove, non era ancora stato pubblicato il nuovo quadro di riferimento, utilizzato per la rilevazione del problem solving nel 2012 (OECD, 2013, pp. 119-137). Bisogna osservare che il nuovo quadro di riferimento di PISA aggiorna quello precedente per due aspetti particolari: la possibilità di far svolgere le prove in formato computerizzato, che permette una maggiore articolazione delle stesse (OECD, 2012a, p. 120); l'enfasi posta sugli aspetti motivazionali ed affettivi come motore per attivare la capacità di risoluzione dei problemi (ibid., p. 123). Quest'ultimo elemento è stato considerato nella costruzione della prova, cercando di partire da situazioni stimolo curiose e potenzialmente interessanti per gli studenti, tutte le prove sono corredate di cartine, mappe, disegni e propongono questioni di tipo personale per un adolescente.

Nella costruzione della prova sono state esaminate ulteriori suggestioni provenienti dalla ricerca (Mayer \& Wittrock, 2006; Jonassen, 2011) e anche indirizzate in modo specifico alla popolazione interessata (Weber, Radu, Mueller, Powell, \& Maher, 2010), considerando da una parte che negli anni della scuola secondaria di I grado si sviluppa la capacità di modellizzazione, legata al passaggio al pensiero astratto, dall'altra che nel problem solving può trovare ampio spazio la creatività (Isaksen, Dorval, \& Treffinger, 2011).

Sono state riprese anche le indicazioni provenienti da un documento «storico», i programmi della scuola media del 1979 (Bellezza, 1979), che 
forse meglio delle diverse edizioni delle Indicazioni nazionali più recenti tracciano un quadro disciplinare chiaro da cui incrociare le abilità di ambito geografico utili per affrontare i tre diversi compiti problematici previsti da PISA (Asquini, 2006, p. 93): prendere decisioni (per esempio scegliendo il percorso più corto su una cartina); analizzare e progettare sistemi (per esempio fornendo informazioni per raggiungere un luogo); localizzare disfunzioni (per esempio incrociando informazioni diverse per escludere alcune opzioni).

Per permettere un confronto con dati di un'altra indagine si è deciso di inserire nella prova anche due testi rilasciati del pacchetto problem solving di PISA 2003 (OECD, 2004, pp. 17 e 73) basati sulla lettura ed interpretazioni di una mappa di trasporti e di una cartina stradale. Nel complesso i testi proposti sono stati 7 , con 24 domande complessive, di cui 6 a scelta multipla e 18 a risposta aperta, univoca o articolata, in cui lo studente deve o scrivere per esteso una risposta o disegnare su una cartina (un percorso o altri elementi). Tra le domande aperte 6 prevedono due livelli di correttezza delle risposte, per cui il numero totale di item che compongono la prova è 30 .

Vista la presenza di domande aperte è stato predisposto uno specifico strumento per la codifica, sulla scorta di quello utilizzato nell'indagine PISA, utilizzato per la trasformazione delle risposte fornite dagli studenti in punteggi (Asquini, 2014a).

Dopo lo svolgimento di un field trial, che ha permesso anche di migliorare l'efficacia dei disegni contenuti nella prova e di verificare la durata effettiva della somministrazione

Lo studio principale si è svolto nel mese di maggio 2014 ed ha coinvolto 1235 studenti di 59 classi terze (due studenti sono stati scartati successivamente per mancate risposte alla prova, quindi un totale di 1233 rispondenti), suddivisi in 9 scuole secondarie di I grado della provincia di Roma.

Successivamente si è deciso di utilizzare la stessa prova su un altro gruppo di studenti, questa volta appartenenti alla seconda classe di scuola secondaria di II grado. Questa seconda somministrazione si è svolta nel mese di maggio 2015 ed ha coinvolto 605 studenti, presenti in 29 classi di 4 licei (2 di Roma, 2 di Latina).

\subsection{Un approfondimento sugli strumenti di misura empirici}

Il presente approfondimento di ricerca sfrutta le informazioni raccolte con alcune domande specifiche poste al termine del fascicolo della prova, sul modello di quanto fatto da PISA nelle ultime edizioni. Questo per verificare a caldo alcuni elementi, in genere riguardanti aspetti tecnici della prova o un'autovalutazione. Nel nostro caso sono state inserite 3 domande riguar- 
danti: l'indicazione dei testi o delle domande risultate più difficili; una autovalutazione della studente sulla prova stessa; l'indicazione degli strumenti utilizzati per calcolare le distanze (attività necessaria in quasi tutte le prove). Per quest'ultima domanda bisogna aggiungere che nelle istruzioni lette in apertura di ogni somministrazione in classe era spiegato che non si potevano utilizzare righelli o squadre (anche perché, come verificato nel corso di tutte le somministrazioni, la disponibilità di questi strumenti non era prevedibile $\mathrm{e}$ si è rivelata effettivamente troppo diversa da classe a classe).

Fra le diverse formae mentis complesse, l'intelligenza visuo-spaziale può essere collegata in modo chiaro alle capacità di risoluzione problemi (Gonzalez, Thomas, \& Vanyukov, 2005; Bühner, Kröner, \& Ziegler, 2008) e conseguentemente anche la capacità di utilizzare strumenti utili per misurare gli spazi può rappresentare un vantaggio per risolvere problemi di ambito geografico.

I dati relativi alla terza domanda circa l'uso di strumenti, è stata pertanto utilizzata per verificare un'ipotesi specifica di ricerca: la quantità e la qualità degli strumenti più o meno empirici utilizzati per risolvere un problema possono essere collegate alla capacità di problem solving.

Per la verifica dell'ipotesi sono stati dunque incrociati i punteggi degli studenti con le risposte alla terza domanda finale, sia considerando il numero di strumenti utilizzati (non c'erano vincoli nella risposta e ogni studente potevano indicarne uno o più), sia la loro tipologia. Nella Tabella 2 sono riepilogati gli strumenti dichiarati dagli studenti per calcolare spazi e distanze nella prova.

Tabella 2. - Strumenti utilizzati dagli studenti.

1. Occhio

2. Dita

3. Penna, matita

4. Altri oggetti

5. Confronto reale-immaginazione

6. Dati della prova

7. Intuito

8. Caso

9. Ragionamento

Come vedremo c'è una forte disparità nelle frequenze di ricorso a tali strumenti, ed è possibile ipotizzare che alcuni di questi strumenti, più o meno empirici, si sovrappongano (il 5 e il 9, o anche il 7 e l'8) e potrebbero essere accorpate, ma in questa prima fase di analisi si è preferito mantenere le categorie indicate dagli studenti (le voci indicate provengono esattamente dalle loro risposte). 
L'analisi è stata svolta sia considerando l'insieme dei due gruppi di rispondenti (secondaria di I grado e di II grado), sia distinguendo i due livelli scolastici, per verificare una prima sottoipotesi: non risultano differenze significative, circa la relazione fra strumenti utilizzati e risultati nel problem solving, fra i due livelli scolastici esaminati.

Infine i dati sono stati analizzati considerando anche l'articolazione per genere, con una seconda sottoipotesi: non risultano differenze significative, circa la relazione fra strumenti utilizzati e risultati nel problem solving, fra maschi e femmine.

\section{I RISULTATI DELL'APPROFONDIMENTO DI RICERCA}

Prima di presentare i risultati specifici dell'approfondimento di ricerca, qualche informazione sui risultati complessivi della prova ${ }^{2}$. Sono state svolte due analisi distinte per i due gruppi esaminati, la prova è risultata consistente per entrambi, con un alfa di Cronbach di 0,781 per la secondaria di I grado e di 0,765 per quella di II grado. I risultati dell'item analisi, svolta con la modalità IRT (Item Response Theory) a due parametri, confermano che tutti gli item, per entrambe le popolazioni, sono risultati efficaci, con valori di difficoltà ben distribuiti lungo la scala e di discriminatività (punto biseriale) sempre superiori a 0,25 . Nel complesso la prova è risultata più difficile per gli studenti della secondaria di I grado, con una media di risposte di 11,02 su 30, mentre per i colleghi delle superiori la media è salita a 14,12. Le due deviazioni standard sono rispettivamente di 4,72 e 4,71, che però rapportate ai punteggi medi denotano una maggiore eterogeneità nella secondaria di I grado, con un coefficiente di variazione di 0,43 , contro lo 0,33 delle superiori. Nel complesso quindi la prova risulta efficace per stimare il tratto latente esaminato, e la sua utilizzazione può essere prevista per entrambe le popolazioni in cui è stata somministrata.

\subsection{I risultati del problem solving e gli strumenti di misura utilizzati}

Per prima cosa vediamo in Tabella 3 come si sono distribuiti gli studenti circa l'uso degli strumenti empirici. È importante premettere che il 7,5\% degli studenti delle medie e il 2,1 delle superiori non hanno risposto alla domanda sugli strumenti. Le percentuali indicate sono relative all'intero numero degli studenti, e ricordiamo che era possibile indicare più di uno strumento.

\footnotetext{
2 Il rapporto di ricerca con tutti i risultati dell'indagine è in corso di pubblicazione.
} 
Tabella 3. - Distribuzione nell'uso degli strumenti (\%).

\begin{tabular}{lccc}
\hline & $\begin{array}{c}\text { Entrambe } \\
\text { LE POPOlAzioni }\end{array}$ & $\begin{array}{c}\text { Secondaria } \\
\text { I Grado }\end{array}$ & $\begin{array}{c}\text { Secondaria } \\
\text { II Grado }\end{array}$ \\
\hline 1. Occhio & 32,6 & 31,9 & 34,2 \\
2. Dita & 23,2 & 23,6 & 22,5 \\
3. Penna, matita & 22,0 & 20,4 & 25,5 \\
4. Altri oggetti & 1,6 & 1,7 & 1,3 \\
5. Confronto reale-immaginazione & 4,7 & 4,1 & 6,0 \\
6. Dati della prova & 14,9 & 15,3 & 13,9 \\
7. Intuito & 3,7 & 3,0 & 5,1 \\
8. Caso & 1,0 & 1,2 & 0,7 \\
9. Ragionamento & 16,5 & 16,9 & 15,9 \\
\hline
\end{tabular}

Una prima osservazione si può fare sul grado di empiricità degli strumenti utilizzati. La prevalenza di quelli concreti, dalle dita ad altri oggetti, è netta, con uno scarto positivo apprezzabile per gli studenti delle superiori circa l'uso della penna come righello improvvisato. Lo strumento più utilizzato però resta l'occhio, considerato affidabile per un calcolo empirico che non richiede una precisione particolare. Il livello di serietà con cui gli studenti hanno affrontato la prova è testimoniato anche dalla scarsa incidenza di «Intuito» e "Caso» come strumenti di misura, mentre costituiscono un indizio di approccio poco empirico gli strumenti 5, 6 e 9, che in diversi modi rimandando ad una elaborazione intellettuale della misura, o cercando di rappresentarla visivamente nella realtà o cercando conforto nei "Dati della prova», come si insegna spesso a scuola, o ragionandoci sopra per vedere se i conti tornano. Come già indicato l'unica differenza netta fra i due livelli scolastici riguarda l'uso della penna.

Ma usare uno strumento o non usarlo, ha una relazione con i risultati della prova? Per alcuni strumenti la risposta è positiva. In particolare chi usa "Penna, matita» raggiunge punteggi significativamente più alti di chi non li usa, mentre per le "Dita" si verifica una netta differenze fra i due livelli scolastici (Tabella 4). Ricordiamo che i punteggi IRT sono stati calcolati su base 500, distintamente per i due gruppi, secondo il modello PISA, e che il confronto è fatto solo per i rispondenti alla domanda sugli strumenti (per entrambe le popolazioni i non rispondenti hanno ottenuto punteggi nettamente inferiori alla media). 
Tabella 4. - Differenze di punteggi per l'utilizzazione degli strumenti.

\begin{tabular}{|c|c|c|c|}
\hline & & $\begin{array}{c}\text { SECONDARIA } \\
\text { I GRADO }\end{array}$ & $\begin{array}{c}\text { SECONDARIA } \\
\text { II GRADO }\end{array}$ \\
\hline \multirow{3}{*}{ 2. Dita } & Utilizzate & 525,4 & 505,3 \\
\hline & Non utilizzate & 500,7 & 500,5 \\
\hline & Sign. &, 001 &, 660 \\
\hline \multirow{3}{*}{ 3. Penna, matita } & Utilizzate & 547,1 & 545,8 \\
\hline & Non utilizzate & 495,7 & 486,1 \\
\hline & Sign. &, 000 &, 000 \\
\hline \multirow{3}{*}{ 9. Ragionamento } & Utilizzate & 476,1 & 456,9 \\
\hline & Non utilizzate & 513,9 & 510,2 \\
\hline & Sign. &, 000 &, 000 \\
\hline
\end{tabular}

Lo strumento empirico immediatamente disponibile, se utilizzato, può fare la differenza per migliorare il risultato nel problem solving, ma con l'eccezione delle "Dita» per gli studenti delle superiori, che non traggono particolari vantaggi dal loro uso. Il netto vantaggio di oltre 50 punti conseguito per entrambi gli utilizzatori di "Penna, matita» dimostra che la capacità di adattare strumenti risulta un efficace requisito per risolvere problemi. Al contrario affrontare un problema pratico come il calcolo di distanze con il semplice «Ragionamento» non aiuta, anzi i risultati diventano significativamente peggiori rispetto a chi utilizza altri tipi di strumenti. Segnaliamo di passaggio che anche gli utilizzatori del "Caso», e dell' "Intuito" per le superiori, ottengono punteggi significativamente più bassi di chi non li usa, ma per questi due strumenti la prudenza è d'obbligo, visto il relativo basso numero di studenti che li utilizzano.

\subsection{I risultati del problem solving e il numero degli strumenti utilizzati}

Nell'ipotesi di questo approfondimento si faceva riferimento non solo alla qualità, ma anche alla quantità degli strumenti utilizzati, considerando che per la domanda esaminata gli studenti erano liberi di indicare uno o più strumenti. La Tabella 5 riepiloga le percentuali degli studenti, anche suddivisi per livello, in relazione a quanti strumenti hanno utilizzato per il calcolo delle distanza. 
Tabella 5. - Distribuzione nel numero di strumenti utilizzati (\%).

\begin{tabular}{lccc}
\hline & $\begin{array}{c}\text { ENTRAMBe } \\
\text { LE POPOLAZIONI }\end{array}$ & $\begin{array}{c}\text { SeCONDARIA } \\
\text { I GRADO }\end{array}$ & $\begin{array}{c}\text { SeCONDARIA } \\
\text { II GRADO }\end{array}$ \\
\hline Non indicato & 5,7 & 7,5 & 2,1 \\
Un solo strumento & 70,1 & 68,6 & 73,1 \\
Due strumenti & 22,4 & 22,4 & 22,5 \\
Tre strumenti & 1,8 & 1,5 & 2,3 \\
\hline Totale & 100,0 & 100,0 & 100,0 \\
\hline
\end{tabular}

Come si può notare circa tre quarti degli studenti utilizza un solo strumento (o non ne utilizza affatto), mentre poco meno di un quarto, con una leggera prevalenza per gli studenti delle superiori, ne utilizza due o tre. Per prima cosa è stata verificata la possibile correlazione fra punteggio e numero di strumenti utilizzati, come indicato in Tabella 6 , distintamente per le due popolazioni.

Tabella 6. - Correlazioni fra punteggio e numero strumenti utilizzati.

\begin{tabular}{clcc}
\hline & & \multicolumn{2}{c}{ Numero strumenti } \\
\hline & & Sec. I grado & Sec. II grado \\
\hline Punteggio & Pearson correlation &, $230\left(^{* *}\right)$ &, $136\left(^{* *}\right)$ \\
IRT500 & Sign. (2-tailed) &, 000 &, 001 \\
& $\mathrm{~N}$ & 1233 & 605 \\
\hline
\end{tabular}

Note: ${ }^{* *}$ correlation is significant at the 0,01 level (2-tailed).

Esiste quindi, per entrambe le popolazioni, una relazione fra i due elementi considerati, per cui è opportuno approfondire il verso di questa relazione, ipotizzando che una maggiore flessibilità nell'uso degli strumenti, attestata dall'indicazione di più di uno strumento, comporti risultati migliori. Notiamo però che la correlazione risulta più forte $(, 230$ vs., 136$)$ per la scuola di I grado. Per verificare sia l'andamento della relazione, sia le probabili differenze fra le due popolazioni, è stata svolta una procedura di confronto delle medie di punteggio secondo il numero di strumenti utilizzati, ed i risultati sono riassunti nella Tabella 7. 
Tabella 7. - Differenze di punteggi per il numero di strumenti utilizzati (\%).

\begin{tabular}{|c|c|c|c|}
\hline & $\begin{array}{c}\text { ENTRAMBE } \\
\text { LE POPOLAZIONI }\end{array}$ & $\begin{array}{l}\text { SECONDARIA } \\
\text { I GRADO }\end{array}$ & $\begin{array}{l}\text { SECONDARIA } \\
\text { II GRADO }\end{array}$ \\
\hline Non indicato & 404,3 & 403,3 & 411,4 \\
\hline Un solo strumento & 497,8 & 499,4 & 494,6 \\
\hline Due strumenti & 524,3 & 525,2 & 522,3 \\
\hline Tre strumenti & 554,8 & 579,0 & 522,0 \\
\hline Sign. &, 000 &, 000 &, 002 \\
\hline
\end{tabular}

Le differenze risultano sempre significative e con un andamento preciso: al crescere del numero di strumenti, cresce il punteggio ottenuto. L'unica particolarità riguarda il livello secondario superiore, dove la differenza si limita all'uso di più di uno strumento, con gli studenti che utilizzano due e tre strumenti che raggiungono lo stesso risultato. Per il livello della secondaria di I grado se è vero che tre strumenti sono stati utilizzati solo dall' $1,5 \%$ degli studenti, la differenza di oltre 50 punti rispetto a chi ne utilizza due è netta, anche verificando l'intervallo di confidenza fra le due misure.

\subsection{I risultati del problem solving per genere}

Per verificare l'ultima ipotesi di ricerca di questo approfondimento sono stati considerate le differenze di punteggio medie per il numero di strumenti utilizzati distinti per genere. Nella Tabella 8 sono riepilogati i risultati dell'analisi per genere.

Tabella 8. - Differenze di punteggi per numero di strumenti utilizzati e per genere.

\begin{tabular}{|c|c|c|c|c|c|c|}
\hline & \multicolumn{2}{|c|}{$\begin{array}{c}\text { ENTRAMBE } \\
\text { LE POPOLAZIONI }\end{array}$} & \multicolumn{2}{|c|}{$\begin{array}{l}\text { SECONDARIA } \\
\text { I GRADO }\end{array}$} & \multicolumn{2}{|c|}{$\begin{array}{l}\text { SECONDARIA } \\
\text { II GRADO }\end{array}$} \\
\hline & Femmine & Maschi & Femmine & Maschi & Femmine & Maschi \\
\hline Non indicato & 395,5 & 414,0 & 397,9 & 408,9 & 381,5 & 459,1 \\
\hline Un solo strumento & 483,5 & 511,3 & 485,7 & 512,5 & 479,1 & 509,0 \\
\hline Due strumenti & 505,5 & 542,5 & 514,0 & 537,0 & 484,6 & 551,8 \\
\hline Tre strumenti & 544,8 & 561,3 & 560,8 & 592,2 & 519,1 & 523,6 \\
\hline Tutti & 484,0 & 514,0 & 486,7 & 511,6 & 478,2 & 518,8 \\
\hline Sign. &, 000 &, 000 &, 000 &, 000 &, 002 &, 002 \\
\hline
\end{tabular}


Il confronto per genere sulla relazione fra risultati nella prova e strumenti utilizzati risulta essere molto stimolante, con andamenti distinti fra i due livelli scolastici esaminati. Per la secondaria di I grado l'andamento è simile fra maschi e femmine, con una crescita progressiva dei punteggi secondo il numero di strumenti e, nel complesso, una differenza di 25 punti a favore dei maschi. Lo scarto fra i due sessi si amplia a 40 punti per gli studenti del secondo anno delle superiori, ma soprattutto, per quello che interessa in questo approfondimento, si modifica l'andamento dei punteggi fra i due sessi: per le femmine non risultano differenze rilevanti fra l'uso di uno o due strumenti, e lo scarto netto è solo a favore di chi ne usa tre; per i maschi si conferma l'andamento complessivo visto per la scuola superiore in Tabella 7 , con un vantaggio che scatta a partire dal secondo strumento utilizzato e con il risultato migliore ottenuto non dagli studenti che utilizzano tre strumenti, ma che ne utilizzano due. C'è da aggiungere che la verifica degli intervalli di confidenza non denota differenze significative fra due e tre strumenti, ma il picco raggiunto dai due strumenti meriterà di essere approfondito.

Le nette differenze delle medie di punteggio fra maschi e femmine, in crescita con il livello scolastico, risultano essere molto più ampie di quelle rilevate da PISA per il nostro paese: nel 2012 il vantaggio dei maschi era di 18 punti (OECD, 2014, p. 182), mentre nel 2003 non risultavano differenze significative, pur con un vantaggio delle femmine di 4 punti (Asquini, 2006, p. 119). Considerando che il dato OCSE ha visto lo stesso passaggio da una differenza non significativa fra i generi nel 2003, a una significativa a favore dei maschi nel 2012 (ma contenuta a 7 punti della scala PISA), sarà necessario approfondire da una parte la relazione specifica tra i risultati per genere dell'indagine sul problem solving geografico e quelli del problem solving in PISA, dall'altra l'evoluzione delle differenze fra i due livelli scolastici.

La relativa mancanza di spazio per questo approfondimento non permette di entrare nel dettaglio circa le possibili differenze esistenti nei singoli strumenti per il genere. La tematica sarà comunque approfondita in tempi successivi.

\section{CONCLUSIONI}

L'analisi dei risultati ha permesso il controllo delle ipotesi formulate per questo approfondimento di ricerca.

La quantità e la qualità degli strumenti più o meno empirici utilizzati per risolvere un problema possono essere collegate alla capacità di problem solving. L'ipotesi risulta verificata sia per il numero di strumenti utilizzati, che 
risulta collegato al risultato rilevato nella prova di problem solving geografico proposta, sia per alcuni degli strumenti specifici, in particolare quelli di tipo empirico utilizzati per stimare in modo sufficientemente precise le distanze sulla carta.

Non risultano differenze significative, circa la relazione fra strumenti utilizzati e risultati nel problem solving, fra i due livelli scolastici esaminati. L'ipotesi risulta parzialmente verificata, poiché se da una parte per la scuola secondaria di I grado la prima ipotesi risulta verificata, per la secondaria di II grado la correlazione, pur significativa, risulta più debole e nel riscontro con i punteggi alcuni strumenti e il numero degli strumenti non sempre risultano collegabili ai punteggi ottenuti nella prova.

Non risultano differenze significative, circa la relazione fra strumenti utilizzati e risultati nel problem solving, fra maschi e femmine. Anche in questo caso l'ipotesi risulta parzialmente verificata, poiché mentre per la somma delle popolazioni e per gli studenti più giovani il legame fra numero di strumenti e punteggi risulta confermato, per la scuola secondaria superiore in modo simile per entrambi i generi, gli andamenti della scuola secondaria superiore sono diversi fra maschi e femmine, anche rispetto all'andamento specifico della popolazione.

Nel complesso quindi una buona capacità di utilizzazione di strumenti in modo creativo alla situazione problematica da risolvere risulta essere utile per ottenere un buon risultato nel problem solving, ma tale capacità varia con l'età e il livello scolastico frequentato e fra i due generi. È doveroso ricordare che la popolazione base dello studio originale era quella della scuola secondaria di I grado, per la quale tutte le ipotesi sono state verificate, a dimostrazione che è in questa fascia di età che è più importante intervenire per migliorare le capacità di risoluzione dei problemi. Un percorso didattico finalizzato a questo obiettivo, come previsto anche dalla normativa scolastica nazionale, può considerare con attenzione i risultati di questo studio, cercando di stimolare da una parte un corretto ricorso agli strumenti empirici per affrontare situazioni problematiche, dall'altra un approccio flessibile e creativo che miri a valorizzare gli strumenti di cui effettivamente si dispone (Perricone \& Polizzi, 2010).

Il fatto che la prova utilizzata in questo studio sia stata di ambito geografico diventa inoltre un esempio di come il problem solving possa trovare spazio in tutte le discipline scolastiche, poiché rappresenta un effettivo approccio trasversale allo studio, che può mettere in moto, ai fini dell'apprendimento, l'ampio spettro di intelligenze di cui dispone ogni essere umano.

La discreta quantità di dati raccolti nel progetto "Problem solving e abilità geografiche. Come valutare la competenza nel triennio della scuola secondaria di I grado» permetterà di compiere ulteriori analisi e impostare 
nuove indagini, soprattutto di tipo qualitativo all'interno delle classi, per indagare le dinamiche cognitive che presiedono le diverse fasi del problem solving, tra cui quella relativa all'uso degli strumenti, nonché le diversità rilevate fra i due livelli scolastici indagati, e in particolare l'evoluzione delle differenze di genere.

\section{RIFERIMENTI BIBLIOGRAFICI}

Asquini, G. (2006). La capacità di Problem Solving dei quindicenni. In M. T. Siniscalco (a cura di), Il livello di competenza dei quindicenni italiani in matematica, lettura, scienze e Problem Solving. Rapporto nazionale di PISA 2003. Roma: Armando.

Asquini, G. (2014a). Definizione di un procedura di codifica delle domande aperte basta sui modelli delle indagini internazionali. Educational, Cultural and Paychological Studies, 10, 463-483.

Asquini, G. (2014b). Lo strano caso dei risultati italiani di PISA 2012. Italian Journal of Educational Research, 7(13), 13-28.

Bellezza, G. (1979). Geografia. In A. Visalberghi et al., Scuola media e nuovi programmi. Firenze: La Nuova Italia.

Bransford, J. D., Brown, A. L., \& Cockling, R. R. (Eds.). (1999). How people learn: Brain, mind, experience, and school. Washington, DC: National Academy Press.

Bühner, M., Kröner, S., \& Ziegler, M. (2008). Working memory, visual-spatial intelligence and their relationship to problem-solving. Intelligence, 36(4), 672680.

De Young, C. G., Flanders, J. L., \& Peterson, J. B. (2008). Cognitive abilities involved in insight problem solving: An individual differences model. Creativity Research Journal, 20(3), 278-290.

Gardner, H. (1983). Formae mentis. Saggio sulla pluralità della intelligenza. Milano: Feltrinelli.

Gonzalez, C., Thomas, R. P., \& Vanyukov, P. (2005). The relationships between cognitive ability and dynamic decision making. Intelligence, 33(2), 169-186.

Greiff, S., Holt, D. V., \& Funke, J. (2013). Perspectives on problem solving in educational assessment: Analytical, interactive, and collaborative problem solving. The Journal of Problem Solving, 5(2), 71-91.

Isaksen, S. G., Dorval, K. B., \& Treffinger, D. J. (2011). Creative approaches to problem solving: A framework for innovation and change. Los Angeles: Sage.

Jonassen, D. H. (2011). Learning to solve problems: A handbook for designing problemsolving learning environments. New York: Routledge.

Mayer, R. E. (1992). Thinking, problem solving, cognition (2nd ed.). New York: Freeman. 
Mayer, R. E. (1998). Cognitive, metacognitive, and motivational aspects of problem solving. Instructional Science, 26, 49-63.

Mayer, R. E., \& Wittrock, M. C. (2006). Problem solving. In P. A. Alexander \& P. H. Winne (Eds.), Handbook of educational psychology (2nd ed.). Mahwah, NJ: Lawrence Erlbaum Associates.

Novick, L. R., \& Bassok, M. (2005). Problem solving. In K. J. Holyoak \& R. G. Morrison (Eds.), The Cambridge handbook of thinking and reasoning. New York: Cambridge University Press.

OECD (1999). Measuring student knowledge and skills: A new framework for assessment. Paris: OECD.

OECD (2003). The PISA 2003 assessment framework. Paris: OECD.

OECD (2004). Problem solving for tomorrow's world. Paris: OECD.

OECD (2013a). PISA 2012 assessment and analytical framework. Paris: OECD.

OECD (2013b). PISA 2015 draft collaborative problem solving framework. https://www. oecd.org/pisa/pisaproducts/Draft\%20PISA\%202015\%20Collaborative\%20 Problem\%20Solving\%20Framework\%20.pdf

OECD (2014). PISA 2012 results: Creative problem solving students' skills in tackling real-life problems (Volume V). Paris: OECD.

OECD (2016). Skills matter: Further results from the survey of adult skills. Paris: OECD.

Perricone, G., \& Polizzi, C. (2010). Valutare gli stili cognitivi nel Problem Solving. Prove di intelligenze applicate e proposte di potenziamento. Trento: Erickson.

Polya, G. (1945). How to solve it. Princeton: Princeton University Press.

Ryjchen, D., \& Salganik, L. H. (2000). Definition and selection of key competencies (De.Se.Co.). Paris: OECD.

Statistics Canada \& OECD (2005). Learning a living: First results of the adult literacy and life skills survey. Ottawa - Paris: Minister of Industry Canada OECD.

Weber, K., Radu, I., Mueller, M., Powell, A., \& Maher, C. (2010). Expanding participation in problem solving in a diverse middle school mathematics classroom. Mathematics Education Research Journal, 22(1), 91-118.

\section{RiassUNTO}

Il saggio presenta un approfondimento dell'indagine "Problem solving e abilità geografiche. Come valutare la competenza nel triennio della scuola secondaria di I grado". Dopo l'indagine principale è stata realizzata anche una rilevazione, con gli stessi strumenti, nella seconda classe della scuola secondaria di II grado, quindi i risultati delle due popolazioni sono comparabili. L'approfondimento mira a indagare il rapporto fra l'uso di strumenti

\footnotetext{
ECPS Journal - 14/2016

http://www.ledonline.it/ECPS-Journal/
} 
empirici per misurare le distanze su carta e i risultati nel problem solving. Viene presentato il modello di problem solving utilizzato da OCSE-PISA anche in relazione alla sua potenziale spendibilità didattica. L'analisi dei dati conferma la correlazione positiva fra l'uso di strumenti e la performance per gli studenti della secondaria di I grado, senza differenze significative per il genere. Più complessa la situazione degli studenti più grandi: l'uso di alcuni strumenti non risulta incidere sui risultati; esistono differenze di genere circa l'andamento dei punteggi per il numero di strumenti utilizzati.

Parole chiave: Geografia, OCSE-PISA, Problem solving, Scuola secondaria, Strumenti empirici.

How to cite this Paper: Asquini, G., \& Marano, F. (2016). Usare strumenti empirici per risolvere problemi: indagine nella scuola secondaria [Using empirical tools to solve problems: A survey in secondary school]. Journal of Educational, Cultural and Psychological Studies, 14, 109-126. doi: 10.7358/ecps-2016-014-asqu 